\title{
El contexto ecológico de las vedas forestales en Michoacán durante el gobierno del General Lázaro Cárdenas 1934-1940
}

\section{The ecological context of forest closures in Michoacán during the government of General Lázaro Cárdenas 1934-1940}

\author{
Víctor Manuel Pérez Talavera* \\ ARCHIVO GENERAL E HISTÓRICO DEL PODER EJECUTIVO DE MICHOACÁN \\ https://doi.org/10.22370/pe.2019.7.2616
}

\section{RESUMEN}

El siguiente trabajo aborda el contexto ecológico en que se restringieron diversas zonas forestales del Estado de Michoacán en México, esto en la década de los treinta del siglo XX durante el periodo de gobierno del General Lázaro Cárdenas del Río (1934-1940) quien se caracterizó por fomentar en el país una política de protección y de sustentabilidad de los bosques. Para el caso de Michoacán la administración cardenista tomó acciones específicas para conservar las áreas boscosas que enfrentaban mayor riesgo de devastación, y debido a su función ecológica y económica era importante proteger, como fue el caso de las regiones de Pátzcuaro, Morelia, Zitácuaro, Uruapan y Angangueo.

Palabras Clave: Michoacán, bosques, deforestación, vedas forestales.

\begin{abstract}
Abstrac
The following work addresses the ecological context in which various forest areas of the State of Michoacán in Mexico were restricted, this in the 1930s during the period of government of General Lázaro Cárdenas del Río (1934-1940) who was characterized for promoting in the country a policy of protection and sustainability of forests. In the case of Michoacán, the Cardenista administration took specific actions to conserve the forested areas that faced the greatest risk of devastation, and due to their ecological and economic function it was important to protect, as was the case in the regions of Pátzcuaro, Morelia, Zitácuaro, Uruapan and Angangueo.
\end{abstract}

Keywords: Michoacán, forests, deforestation, forest closures.

*vic_aute@hotmail.com 


\section{LA APLICACIÓN DE VEDAS FORESTALES}

Las vedas que se aplicaron durante el periodo cardenista tuvieron como finalidad la preservación de los recursos forestales, sobre todo en aquellos lugares en donde la explotación de éstos representaba un severo problema en el desequilibrio ecológico. Ante esta problemática, el gobierno implementó diversos mecanismos legales para tratar de detener la deforestación en la entidad. Fue a partir de la creación de decretos presidenciales que se dispuso la protección de diversas zonas boscosas en la entidad. Sin embargo, esta sería de disposiciones conllevaba a una serie de requisitos que había que cubrir. Por ejemplo, el gobierno federal tenía que determinar mediante diversos estudios técnicos las condiciones en que se encontraban los predios que se someterían a revisión, es decir, analizar las características del terreno y los recursos forestales que contenía la superficie. ${ }^{1}$ Asimismo, era importante escuchar la opinión de los pobladores y de las autoridades locales sobre los decretos que se aplicarían en una determinada zona, (incluidos los ejidatarios y comuneros). Otro aspecto importante que se tomó en consideración fue la justificación técnica y ecológica que se realizaba sobre el área que sería vedada, ya que ésta debía de contar con un estudio previo que reflejara las repercusiones económicas y sociales que la medida tendría en un futuro a través de un programa de evaluación y seguimiento, sobre el impacto que tendría la restricción de los bosques en las comunidades donde se dependía en un alto porcentaje del aprovechamiento de éstos. ${ }^{2}$

Igualmente, los decretos debían de especificar el tiempo de duración, así como los usos y actividades que se permitirían en cada predio y las prohibiciones de que serían objeto. Uno de los puntos que más preocupó a las poblaciones en donde serían aplicadas las disposiciones sobre la suspensión de bosques, fueron las alternativas económicas que se generarían, teniendo en cuenta que una de las principales fuentes de trabajo en las zonas rurales era precisamente la explotación forestal. El gobierno estaba obligado a brindar a las comunidades diversas alternativas de desarrollo rural. En su 
momento la administración cardenista priorizó la resinación como fuente de trabajo para favorecer la economía local. La conformación de cooperativas resineras a cargo de los mismos habitantes fue el medio mediante el cual el Ejecutivo organizó a los pobladores para dicho fin. ${ }^{3}$

Para cumplir con todos los aspectos mencionados anteriormente, era de vital importancia que hubiera una estrecha participación tanto de las autoridades forestales como de la población en general; los primeros se encargarían de hacer cumplir la ley, y los segundos de respetarla. Cabe mencionar que las vedas forestales tenían fundamento constitucional a través de la Ley Forestal de 1926, especialmente en el artículo 41 que establecía que la Secretaría de Agricultura y Fomento quedaba a cargo de todas las medidas necesarias para el cumplimiento de la ley, así como de formar todos los reglamentos así como de dictar las disposiciones especiales conducentes a conservar los bosques. ${ }^{4}$

El 4 de febrero de 1936 el Departamento Forestal de Caza y Pesca, a cargo de Miguel Ángel de Quevedo, emitió el decreto que declaraba zona protectora forestal vedada los terrenos de la cuenca hidrológica del Lago de Pátzcuaro. Conformado por los municipios de Quiroga, Tzintzuntzan, Erongarícuaro y Pátzcuaro. En la justificación jurídica que se planteó para proteger dicha área influyeron varios factores ecológicos, ya que se consideró la urgencia de proteger las vertientes de la cuenca y de los predios que redondeaban a ésta, debido al crecimiento por el cambio de uso de suelo, además de que los terrenos eran impropios para la agricultura y para la explotación de los bosques.

El gobierno advirtió que de no atenderse dichas recomendaciones a tiempo podrían presentarse riesgos de erosión de suelos. Por lo tanto, era necesario evitar que la deforestación pudiera traer consigo el desprendimiento de los cerros, ya que a falta de la vegetación éstos podían deslavarse, perjudicando al lago al perder éste su fondo y cause con el latente peligro de secarse y desaparecer. El problema de los azolves se veía como una amenaza ya que no solamente reducía el nivel de la profundidad del agua, sino que también traía consigo 
la pérdida de la vegetación que se hallaba en el lago, la cual tenía como función importante generar el alimento para la reproducción del pescado. Éste a su vez representaba la principal dieta de los pobladores vecinos de la ribera del lago de Pátzcuaro, quienes adicionalmente se veían beneficiados con una fuente de trabajo a partir de la pesca que realizaban. Por estas consideraciones es que la autoridad forestal se vio en la necesidad de decretar y suspender toda actividad de explotación en los montes que rodeaban al Lago de Pátzcuaro:

Cerro del Frijol, Loma de la Cantarita o de Huiplo, Malpaís de la Fundición, Cerro de la Cantera, Cerro de San Miguel, Cerro del Tambor, Loma de Ajuno, Loma de Copándaro, Cerro de Huincho, Cerro de Caracatúa, Cerro del Mexteño, Cerro del Conejo, Cerro del Timbre o San Geronimo, Cerro de las Abejas, Cerro Chino, Cerro Azul, Cerro de Sanambu, Cerro del Divisadero o Rancho Seco, Loma de Corrales, Loma de Balastre, Loma Prieta, Loma de la Nopalera, Loma de los Cerritos, Loma de la Venta o Providencia y Cerro de Cuanajo. ${ }^{5}$

En la reglamentación que se estableció para la cuenca de Pátzcuaro quedó prohibida la apertura de terrenos para el cultivo agrícola dentro de la serranía o hacer cambios de uso de suelo en donde hubiera vegetación. La veda también canceló la introducción de ganado en los predios destinados a repoblación del arbolado. De igual manera, en aquellas zonas en donde las tierras presentaran problema de erosión, se dispuso la puesta en práctica de un programa de reforestación a cargo de los propietarios de las tierras en coordinación con del Departamento Forestal, esto con la intención de contrarrestar la erosión de los montes y evitar los azolves al lago. ${ }^{6}$

Para el 8 de septiembre del mismo año, la cuenca hidrológica del río Chiquito en Morelia también entró en la misma dinámica de restricción forestal. Las razones que se argumentaron para decretar la veda de dicha zona fue la incesante deforestación que se estaba 
realizando en perjuicio de los habitantes de la ciudad. El gobierno federal consideró que la vegetación del lugar formaba uno de los principales agentes de retención de las aguas pluviales en terrenos de montańa, dando lugar a efloraciones permanentes de las mismas en forma de manantiales y de corrientes de agua limpia y potable, por tal motivo era necesario que se mantuviera en buenas condiciones para evitar su deforestación y la erosión de las tierras descubiertas, de lo contrario se reduciría el caudal de la corriente, dando origen a la contaminación de las aguas, lo cual representaría un problema grave para los morelianos. Los límites que se fijaron para determinar el área que sería vedada, fueron los siguientes:

Partiendo de la hacienda del Molino hacia al Oriente, hasta llegar al cerro de La Nopalera, pasando por el puerto del Venado hasta terminar en el cerro de Aparicio; de este punto, bajando hacia el Sur, se sigue la línea que une los puntos del cerro Alto con los cerros de Punta de Tierra y La Rosa, cambiando de dirección hacia el Suroeste se continua hasta el cerro Azul siguiendo por los parajes denominados Cruz de Piedra, Puerto de Campanario, Puerto del Sauz, La Lechuguilla, Agua Zarca y La Mora; de este punto y con dirección al Oeste se sigue la línea recta que pasa por la Cruz de los Zimbos o Cruz Gorda y llega al Cerro Verde; de aquí, con rumbo al Noroeste, se llega al Puerto de Zimpanio; volviendo a cambiar de dirección hacia el Noreste se sigue la línea hasta encontrar el puerto del Tejocote Redondo y siguiendo la misma recta se llega al poblado de Jesús del Monte y finalmente de este punto el polígono se cierra encontrando el punto de partida o sea la hacienda del Molino. ${ }^{7}$

Así quedó prohibida por tiempo indefinido la explotación comercial de los bosques existentes en dicha zona. Al mismo tiempo, la autoridad forestal aplicó un programa de reforestación para todos aquellos terrenos que estuvieran deforestados, para evitar deslaves y azolves que pudieran afectar la corriente del río Chiquito 
y que esto pudiera propiciar en un futuro inundaciones. En lo concerniente a los pobladores vecinos que se sintieran afectados en el aprovechamiento del bosque, éstos podían a través de la autorización del Servicio Forestal, tener acceso a los productos forestales que fueran susceptibles de extraerse e indispensables para sus necesidades domésticas. Esta parte de la normativa quedó ambigua ya que no se especificaba claramente algunos conceptos como ¿a qué se referían con la necesidad de aprovechar los recursos forestales únicamente para el uso doméstico? Se puede deducir que quizá se referían únicamente a la madera como uso diario para combustible. Pero también creo que la madera utilizada para construir una vivienda podría ser parte de las necesidades domésticas. En este sentido, no quedó claro o especificado a qué se refería con uso doméstico ya que su interpretación fue general y a criterio de la autoridad.

Igualmente había una restricción en cuanto a la elaboración de productos forestales, pero no se especificaba si eran utensilios de cocina, o si se referían a herramientas para el campo; no se especificaba qué tipo de artículos eran los que se podían elaborar o cuales no estaban permitidos de acuerdo a la norma forestal. Se entiende entonces que estas decisiones quedaban a consideración del Servicio Forestal como parte responsable de vigilar que se aplicara la ley en los municipios en la entidad, por ende eran quienes decidían cómo, dónde y cuándo se podían hacer aprovechamientos de madera en el monte, circunstancia a la que tenían que adaptarse los pobladores.

Para el 14 de octubre de 1936 se protegieron los montes circundantes de la ciudad de Morelia, especialmente los que tenían que ver con la cuenca hidrográfica del río Grande de Morelia debido a que era urgente efectuar diversas medidas de conservación de los bosques que rodeaban dicha cuenca, con la intención de evitar que el acarreo de las tierras de las partes superiores empobreciera las partes bajas, las cuales tenían un potencial para ser utilizadas en trabajos agrícolas o bien para impulsar la ganadería de las zonas cercanas a la capital michoacana. A la administración cardenista le preocupaban las extensas superficies boscosas que se estaban deforestando por 
el uso desmedido del aprovechamiento forestal, debido a diversas actividades que se realizaban en la zona: construcción de casas, extensión de cultivos, elaboración de carbón vegetal, así como el exceso de árboles talados por parte de los pobladores para fines particulares. Estas explotaciones eran consideradas por el gobierno como destructoras del entorno natural, por tanto habría que priorizar el aprovechamiento racional en beneficio de los propios habitantes. Para el caso de la ciudad de Morelia la fracción de linderos que se delimitaron para implementar la veda forestal, fueron los siguientes:

Partiendo del cerro del Correo, en el vértice Noroeste, la línea sigue al Este, pasando por el cerro de El Águila, presa de Undameo, cerro de las Animas, cerro de La Huerta, cerros de la Joya, hasta el cerro Verde; de este lugar, con dirección Sur y Sureste en general, la línea sigue sobre el filo de la serranía cuyas vertientes alimentan con sus aguas al río Grande de Morelia, tocando entre otras la loma de Palo Amarillo, el cerro de Huizota y loma de la Cruz Gorda, hasta el cerro de Tzintzun; de este lugar y con dirección general al Oeste la línea pasa por el cerro Chaparro, cerro de los Barcos, cerro del Timbicho y cerro del Burro, hasta el cerro del Frijol; posteriormente, y con dirección general hacia el Norte, la línea pasa por cerro de La Cal, cerro de La Venta, Loma Prieta, Loma Blanca y cerro del Divisadero para terminar en el cerro del Correo, que se tomó como punto de partida. ${ }^{8}$ 
Mapa i. Veda forestal de los montes cercanos a los ríos Grande y Chiquito de Morelia

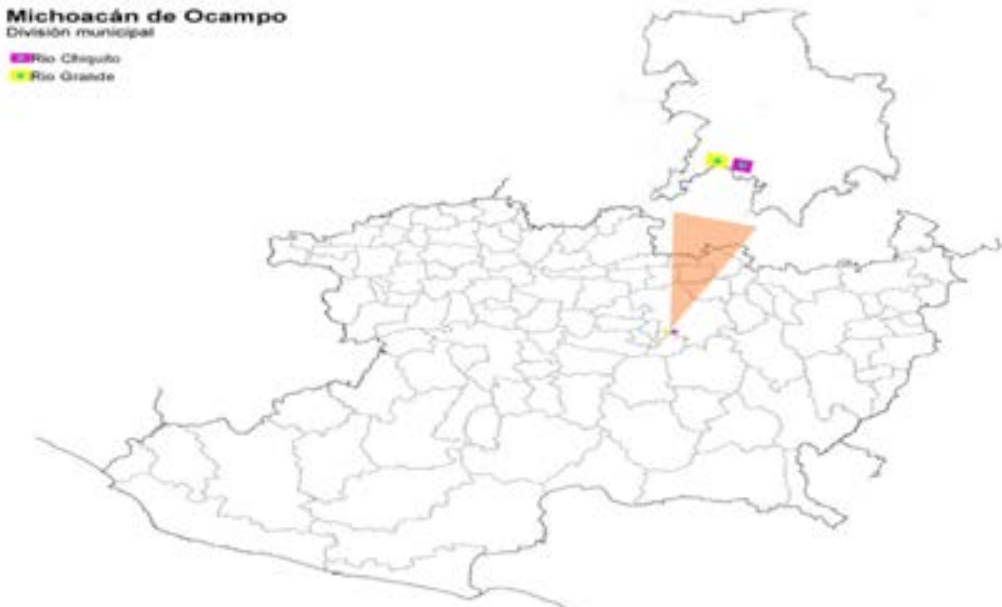

El río Grande quedó vedado por tiempo indefinido y se restringió la explotación de carácter comercial en la zona. En lo concerniente a las localidades vecinas que entraron en el decreto de veda, únicamente les fue autorizado el aprovechamiento de madera de acuerdo a los criterios que determinaba el Servicio Forestal. Para garantizar el cumplimiento de la disposición legal fue necesario que la autoridad se coordinara con las comunidades de la región para crear un amplio sistema de vigilancia, además de un programa de reforestación para repoblar las zonas más afectadas. ${ }^{9}$

El 4 de enero de 1937 se estableció igualmente como zona protectora forestal los terrenos boscosos que rodeaban a la ciudad de Zitácuaro. El gobierno federal como parte de su política conservacionista, consideró necesario vedar los cerros cercanos a dicha ciudad, debido a que se venía incrementando la pérdida del bosque y de la vegetación silvestre en el lugar; esta última desempeñaba una función primordial en términos de protección del suelo, y como reguladora de las condiciones del clima. La justificación que el gobierno argumentó para la suspensión forestal en el lugar, estuvo relacionado con el peligro que representaba la extensión cada 
vez mayor de los terrenos desprovistos de vegetación, lo que traía como consecuencia el arrastre de tierras que azolvaban los causes de los ríos que atravesaban por la serranía de la ciudad. Esto aunado a la topografía del lugar ya que eran terrenos con pendientes muy altas las que rodeaban a la población, situación que ocasionaba en algunos casos severas inundaciones. ${ }^{10}$ Por tal motivo se consideró importante conservar la vegetación arbórea que se encontraba en ella para mantener en buenas condiciones el desarrollo del lugar. La delimitación que se estipuló para suspender las actividades de explotación de los bosques en la región estuvo de la siguiente manera:

Se declara zona protectora forestal vedada la porción de terrenos forestales que rodean a la ciudad de Zitácuaro. Por el Norte, del punto conocido con el nombre de Ojo de Agua de Aranza a Ocurio de Arriba; por el Este, de Ocurio de Arriba a San Isidro; por el Sur, de San Isidro al Bosque; por el Oeste; de El Bosque al Ojo de Agua de Aranza. La superficie toral comprendida es aproximadamente de 13,680 hectáreas. ${ }^{11}$

Al igual que en otros puntos forestales del estado, la explotación de los bosques con fines comerciales quedó estrictamente prohibido, y los pobladores quedaron limitados únicamente a la provisión de madera seca para sus necesidades domésticas. En lo que refería a la parte de la reforestación de los montes de la ciudad de Zitácuaro, se estableció un vivero forestal para que éste pudiera dotar de los árboles suficientes a la región. De igual forma se ordenó que entre los pueblos vecinos, principalmente entre aquellos que habían pedido al gobierno federal una porción de tierras para sus ejidos, se les dotara de una determinada cantidad de árboles para que realizaran plantaciones en las tierras otorgadas. ${ }^{12}$

Cabe mencionar que en la región de Zitácuaro además de implementar medidas de prevención de inundaciones, también se pudo observar una clara idea de preservar los bosques y de repoblar las zonas más afectadas, principalmente en aquellos lugares donde se estaban construyendo carreteras. Cabe mencionar que para 1937 se 
construía la carretera Zitácuaro-Toluca y Zitácuaro-Morelia, estos trabajos de apertura de vías de comunicación terrestre perjudicaron a su paso varias zonas forestales, motivo por el cual se comprendió la necesidad de establecer un vivero para reforestar dicha región. Es pertinente señalar que Miguel Ángel de Quevedo en un informe entregado al presidente Cárdenas en 1936, advirtió sobre la alarmante deforestación que estaba causando la construcción de carreteras, principalmente en el trayecto entre Zitácuaro y Toluca. ${ }^{13}$

En relación a la ciudad de Uruapan, el 17 de febrero de 1937 fueron vedados los montes vecinos que rodeaban dicha localidad. El objetivo que se estableció fue el de reducir los altos índices de explotación forestal que se realizaban en la zona. Asimismo, se tomó en consideración la repoblación del arbolado para proteger los bosques que rodeaban al centro urbano, a fin de evitar el peligro de las degradaciones del suelo y prevenir que en un futuro pudiera traer consigo problemas de inundaciones y de salubridad entre la población. Se priorizó la protección de diversos manantiales que nacían en el lugar debido a la importancia que representaban no sólo para las necesidades de los pobladores de la ciudad, sino también para la fuerza motriz de diversas industrias que la utilizaban, así como para los trabajos agrícolas del valle de Apatzingán, cuyos beneficios eran necesarios para sostener una economía local. La preservación de los bosques en la región se consideró que era de gran importancia debido a los beneficios que traía consigo tanto en el aspecto ecológico como en lo económico. Cabe mencionar que uno de los factores que también determinó la veda en esta zona, fue la necesidad de conservar un área forestal perfectamente protegida contra las explotaciones comerciales, especialmente en los cerros de La Alberca, La Cruz, El Colorado, Las Varas y El Chino. En este contexto el polígono que se delimitó para establecer el decreto de suspensión forestal fue el siguiente:

Por el Norte, del cerro de Cheranguerán al cerro de La Alberca; por el Oriente, del cerro de la Alberca al cerro Colorado, prosiguiendo los linderos hasta Zirimícuaro; por el 
Sur, de Zirimícuaro a la Tzaráracua, y por el Occidente, de la Tzaráracua al cerro Chino, continuando los linderos hasta el punto de origen, o sea el cerro de Cheranguerán. ${ }^{14}$

El área delimitada comprendió una superficie aproximada de 13, 664 hectáreas, en la zona quedó estrictamente prohibida la explotación de los bosques así como el cambio de uso de suelo por cultivos agrícolas o de pastoreo. Asimismo, se estipuló que el Departamento Forestal fuera el encargado de repoblar los montes que se encontraran más afectados y así prevenir deslaves en la ciudad.

Por otra parte el 14 de noviembre de 1940 la Secretaría de Agricultura y Fomento decretó la suspensión de la tala de bosques en el municipio de Angangueo. Es de resaltar que fue una de las últimas vedas forestales que se dieron durante la administración de Lázaro Cárdenas como presidente de México. Dicha disposición se llevó a cabo debido a las constantes quejas de los pobladores de la región por tala que realizaban companías madereras en los alrededores de la localidad, situación que estaba ocasionando el agotamiento de los manantiales en perjuicio de la comunidad. Mediante una política de prevención y protección, el gobierno cardenista implementó una serie de medidas precautorias para evitar que los cuerpos de agua pudieran disminuir. De igual manera se consideró un aspecto importante: la necesidad de conservar la vegetación de los bosques maderables y de los no maderables, es decir, no sólo se requería preservar el arbolado, sino también los arbustos y los pastos que crecían en el lugar como una forma de evitar deslaves en la zona, esto debido a que en la geografía del lugar había una gran cantidad de terrenos con pendientes inclinadas, proclives a derrumbarse. Hay que destacar que fueron dos las razones por las que se decretó la veda en la región: una que tuvo que ver con la protección de la cubierta vegetal para evitar derrumbamiento de cerros que pudieran afectar a los vecinos de la localidad de Angangueo, ${ }^{15}$ y la segunda que se enfocó a cuidar los manantiales que nacían en el lugar y evitar la pérdida de éstos. La veda que se estableció para restringir los bosques en la población de Angangueo se fijó bajo las siguientes referencias: 
Se declara veda indefinida para los bosques comprendidos en los alrededores de la población de Angangueo. Tomando como punto de partida el cerro del Tecolote y Mimbre, localizado al Poniente de la población de Angangueo, Mich., de este punto de sigue con la dirección general hacia el Norte pasando por las cimas de los cerros llamados El Magueyal, Coyote y Mirador; de este punto la línea sigue con una dirección N.E., hasta tocar con el cerro de Las Lagunillas; de este lugar se sigue con dirección Sur hasta el cerro de La Reina; de este punto la línea sigue con una dirección S.E., muy próxima a la línea divisoria entre los Estados de México y Michoacán, tocando los cerros de Catingoncito, El Charro, La Gotera, siguiendo de este lugar al lindero con dirección S.W., tocando los cerros de Las Cruces, Cerro Prieto y La Corona, de aquí con una dirección N.W., hasta tocar el cerro de Amparo, de este punto, con dirección Norte, se llega al cerro Tecolote y Mimbre, que sirvió como punto de partida. ${ }^{16}$

\section{Mapa 2. Vedas forestales establecidas en Michoacán DURANTE LOS AÑOS DE I934-I940}

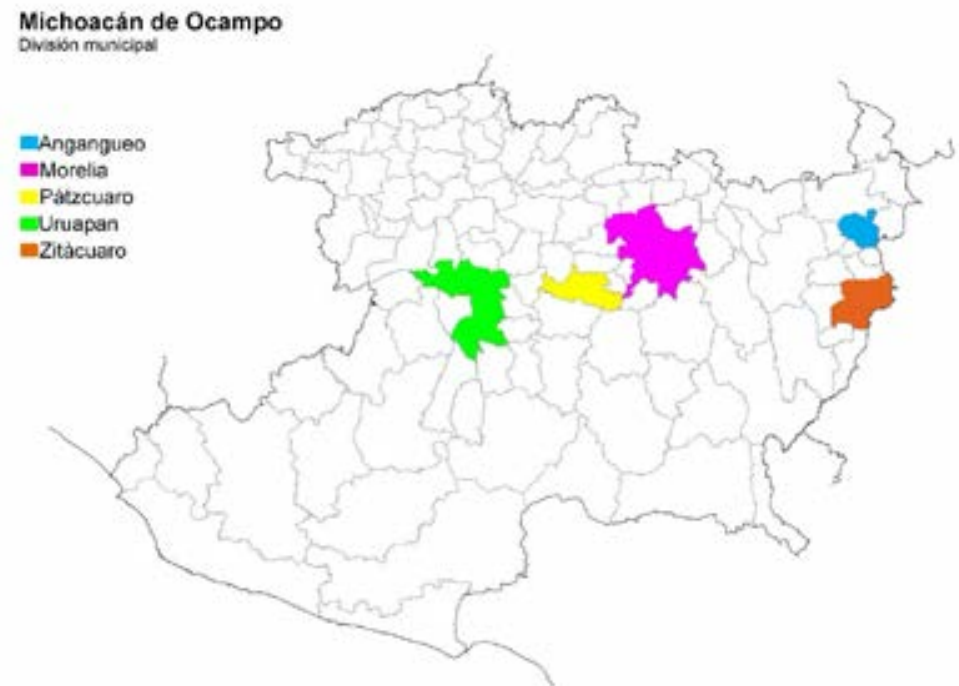


Al igual que en diferentes puntos geográficos del estado de Michoacán en donde se establecieron las vedas forestales durante el periodo cardenista, en Angangueo también se restringió la explotación de los bosques con fines comerciales e industriales, sin embargo, a diferencia de otras regiones, en esta zona se prohibió el cambio de uso de suelo en un tema muy específico que estuvo relacionado con la conformación de las huertas frutícolas. Esta decisión nos da una idea más clara sobre el problema que se enfrentaba ya que había un exceso en la proliferación de tierras de cultivo, las cuales estaban disminuyendo las áreas forestales del lugar. En lo que refiere al aprovechamiento que realizarían los pobladores, únicamente se les permitió la utilización de madera seca para uso diario de combustible.

\section{Conclusiones}

A manera de conclusión se puede decir que las políticas forestales implementadas por el gobierno de Lázaro Cárdenas en el estado de Michoacán, se aplicaron a partir de dos objetivos: primero, dichas disposiciones se basaron en la protección y conservación de las zonas de mayor importancia ecológica, que el gobierno consideró deberían de preservarse para beneficiar no solamente al entorno natural, sino también la salud de los ciudadanos al propiciarles una mejor calidad del medio ambiente. La segunda vertiente se basó en la prevención de desastres naturales, la preocupación que tenía la administración cardenista en torno a la deforestación, no solamente en Michoacán sino en el país, fue por la latente posibilidad de la generación de deslaves, principalmente en aquellas ciudades que estaban rodeadas de montes con elevadas inclinaciones. Un ejemplo de esto fueron los decretos de restricción forestal que se aplicaron en los municipios de Uruapan, Pátzcuaro, Morelia, Zitácuaro y Angangueo localidades que eran altamente propicias a ser afectadas. Si bien es cierto que en cada región de Michoacán las veda forestales se aplicaron de acuerdo a las condiciones y problemáticas que aquejaba a cada zona en particular, estas políticas de prevención tenían un claro objetivo conservacionista. Sin embargo, es importante mencionar 
que dichas disposiciones tuvieron resistencia en diferentes sectores de la sociedad, sobre todo entre la población rural donde generó inconformidad al verse limitados sus derechos al aprovechamiento de los bosques; la autoridad, era quien racionaba a la población de la madera pero únicamente para las necesidades básicas que se utilizaban para combustible.

\section{Fuentes CONSULTADAS}

Archivo General e Histórico del Poder Ejecutivo de Michoacán, Diario Oficial de la Federación, México 24 de abril de 1926. Archivo General e Histórico del Poder Ejecutivo de Michoacán, Diario Oficial de la Federación, México, martes 4 de febrero de 1936.

Archivo General e Histórico del Poder Ejecutivo de Michoacán, Diario Oficial de la Federación, México, martes 8 de septiembre de 1936.

Archivo General e Histórico del Poder Ejecutivo de Michoacán, Diario Oficial de la Federación, México, miércoles 14 de octubre de 1936.

Archivo General e Histórico del Poder Ejecutivo de Michoacán, Diario Oficial de la Federación, México, lunes 4 de enero de 1937.

Archivo General e Histórico del Poder Ejecutivo de Michoacán, Diario Oficial de la Federación, México, miércoles 17 de febrero de 1937.

Archivo General e Histórico del Poder Ejecutivo de Michoacán, Diario Oficial de la Federación, México, jueves 14 de noviembre de 1940.

Archivo General de la Nación Mexicana, Fondo Lázaro Cárdenas del Río, caja 555, expediente 501.1/117, fs. 239-248.

Periódico El Universal, México, 11 de febrero de 2010. 
BiBLIOGRAFÍA

AGUIRRE BELTRÁN, GONZALO, Problemas de la población indigena de la cuenca de Tepalcatepec (Memorias del Instituto Nacional Indigenista), México, Instituto Nacional Indigenista, Tomo III, Volumen III, 1952.

AMEND STEPHAN, GIRALDO AÍDA (et, al), Parques Nacionales y Conservación Ambiental; Planes de Manejo Conceptos y Propuestas, Panamá, Unión Internacional para la Conservación de la Naturaleza, 2002.

BECKER MARJORIE, "El cardenismo y la búsqueda de una ideología campesina", en Relaciones, Zamora, volumen 8, número 29, 1987.

BOYER R. CHRISTOPHER, "El Manejo del Paisaje Social en los Bosques Posrevolucionarios. Una Reinterpretación del Cardenismo", en Eduardo N. Mijangos Díaz, Alonso Torres Aburto (Coordinadores), Colección del Centenario de la Revolución, Morelia, Instituto de Investigaciones Históricas, Facultad de Historia, Universidad Michoacana de San Nicolás de Hidalgo, 2011.

BOYER R. CHRISTOPHER, "Terrenos en disputa. La reglamentación forestal y las respuestas comunitarias en el noroeste de Michoacán. 1940-2000,” en David Bray, Leticia Merino y Deborah Barry (editores), Los bosques comunitarios de México. Manejo sustentable de paisajes forestales, México, Secretaría de Medio Ambiente y Recursos Naturales, Instituto Nacional de Ecología, Instituto de Geografía de la Universidad Nacional Autónoma de México, Consejo Civil Mexicano para la Silvicultura Sostenible, Florida International University, 2007.

BLANCO MOHENO, ROBERTO, Tata Lázaro: vida, obra y muerte de Cárdenas, México, Editorial Diana, 1972.

CALDERÓN MÓlGORA, MARCO ANTONIO, Historias, procesos politicos y cardenismo, Zamora, El Colegio de Michoacán, 2004.

CÁRDENAS LÁZARO: Apuntes. Una selección, México, 
Universidad Nacional Autónoma de México, Centro de Estudios de la Revolución Mexicana, Tomo I, 23 de junio de 1931.

DIETZ GUNTHER, La comunidad purhépecha es nuestra fuerza: etnicidad, cultura y región en un movimiento indigena en Michoacán, México, Quito Ecuador, ediciones Abya-Yala, 1999.

\section{Notas AL FINAL}

1 "Vedas forestales en México", http://biblio.juridicas.unam.mx/ libros/4/1927/9.

2 "Vedas forestales en México", http://biblio.juridicas.unam.mx/ libros/4/1927/9.

3 "Vedas forestales en México", http://biblio.juridicas.unam.mx/ libros/4/1927/9.

${ }^{4}$ AGHPEM, Periódico Oficial de la Federación, Ley Forestal de 1926, México 24 de abril de 1926, pp. 1051- 1055.

${ }^{5}$ AGHPEM, Diario Oficial de la Federación, México, martes 4 de febrero de 1936, p 7.

${ }^{6}$ AGHPEM, Diario Oficial de la Federación, México, martes 4 de febrero de 1936, p 7.

${ }^{7}$ AGHPEM, Diario Oficial de la Federación, México, martes 8 de septiembre de 1936, pp. 10 y 11.

${ }^{8}$ AGHPEM, Diario Oficial de la Federación, México, miércoles 14 de octubre de 1936, pp. 10 y 11.

9 AGHPEM, Diario Oficial de la Federación, México, miércoles 14 de octubre de 1936, pp. 10 y 11.

${ }^{10}$ AGHPEM, Diario Oficial de la Federación, México, miércoles 14 de octubre de 1936, pp. 10 y 11.

${ }^{11}$ AGHPEM, Diario Oficial de la Federación, México, lunes 4 de enero de 1937, pp. 9 - 11.

${ }^{12}$ AGHPEM, Diario Oficial de la Federación, México, miércoles 14 de octubre de 1936, pp. 10 y 11.

${ }^{13}$ AGNM, Fondo Lázaro Cárdenas del Río, caja 555, expediente 501.1/117, fs. 239-248. 
${ }^{14}$ AGHPEM, Diario Oficial de la Federación, México, miércoles 17 de febrero de 1937, pp. 10 y 11.

${ }^{15}$ Esta política preventiva que la administración cardenista implementó de cuidar la vegetación y conservar la reforestación en los montes de Angangueo, nos puede dar una idea más clara del por qué al gobierno le preocupaban los deslaves en la zona. Setenta años después, el día 7 de febrero de 2010, el municipio de Angangueo y comunidades circunvecinas experimentaron uno de los peores desastres naturales de que se tenga registro en la historia del estado de Michoacán. En esa ocasión el desgajamiento de los cerros vecinos a la población se llevó cientos de casas, lo que propició el deceso de más 20 personas y cientos de familias damnificadas que se quedaron sin hogar en el municipio. Las causas, según argumentaron los especialistas en la materia, fueron la tala inmoderada en la zona, así como al cambio de uso de suelo con fines habitacionales y de agricultura. Véase, El Universal, México, 11 de febrero de 2010.

${ }^{16}$ AGHPEM, Diario Oficial de la Federación, México, jueves 14 de noviembre de 1940, pp. 3 y 4.

Fecha de Recepción: 5 mayo de 2018

Fecha de Aceptación: 25 de julio de 2018

VERSIÓn FINAL: 2 de agosto de 2018 